An Infinite Particle System with Additive Interactions

Author(s): Richard Durrett

Source: Advances in Applied Probability, Vol. 11, No. 2 (Jun., 1979), pp. 355-383

Published by: Applied Probability Trust

Stable URL: http://www.jstor.org/stable/1426844

Accessed: 11-06-2015 19:45 UTC

Your use of the JSTOR archive indicates your acceptance of the Terms \& Conditions of Use, available at http://www.jstor.org/page/ info/about/policies/terms.jsp

JSTOR is a not-for-profit service that helps scholars, researchers, and students discover, use, and build upon a wide range of content in a trusted digital archive. We use information technology and tools to increase productivity and facilitate new forms of scholarship. For more information about JSTOR, please contact support@ jstor.org. 


\title{
AN INFINITE PARTICLE SYSTEM WITH ADDITIVE INTERACTIONS
}

\author{
RICHARD DURRETT , * University of California, Los Angeles
}

\begin{abstract}
The models under consideration are a class of infinite particle systems which can be written as a superposition of branching random walks. This paper gives some results about the limiting behavior of the number of particles in a compact set as $t \rightarrow \infty$ and also gives both sufficient and necessary conditions for the existence of a non-trivial translation-invariant stationary distribution.

INFINITE PARTICLE SYSTEM: RANDOM WALK: BRANCHING PROCESS: POINT PROCESS
\end{abstract}

\section{Introduction}

In this paper we shall study a class of Markov processes whose state at each time is a countable (or finite) subset of $R^{d}$. The evolution of these processes may be, informally, described by the following rules. If $\eta_{t}$ is the state at time $t$ then

(i) for any $x \in \eta_{t} P\left\{x \notin \eta_{t+s}\right\}=s+o(s)$,

(ii) there is a non-negative measurable function $b$ such that for any open set $G$ with $G \cap \eta_{t}=\varnothing$

$$
\begin{gathered}
P\left\{\eta_{t+s} \cap G \neq \varnothing\right\}=s\left(\sum_{x \in \eta_{t}} \int_{G} b(x, y) d y\right)+o(s) \\
P\left\{\eta_{t+s} \cap G \text { contains two points }\right\}=o(s) .
\end{gathered}
$$

In the terminology of Holley and Stroock (1978) what we have described is a birth and death process in which the death rate for particles is one (independent of the configuration) and the birth rate at $y$ when the state is $\eta$ is given by $\sum_{x \in \eta} b(x, y)$. In this paper we shall prove some results about the limiting behavior of $\eta_{t}$ as $t \rightarrow \infty$ and we shall use these results to give both sufficient and necessary conditions for the existence of a non-trivial translation-invariant stationary distribution. For most of the results given below we shall assume that the process is spatially homogeneous, that is, $b(x, y)=b(0, y-x)$. In some cases, however, we can obtain conclusions under the weaker assumption that $\int b(x, y) d y=\beta<\infty$ where $\beta$ is a constant (independent of $x$ ). We shall always assume that the second condition is satisfied.

Received 1 August 1977; revision received 30 May 1978.

* Postal address: Department of Mathematics, University of California, Los Angeles CA 90024, U.S.A. 
The key to our analysis is the observation that if the process starts with a finite number of particles then $\eta_{t}(K)$, the number of points in $\eta_{t} \cap K$, has the same distribution as the corresponding quantity in a particle system which operates according to the following rules:

(i) all particles have exponential lifetimes with mean $1 /(\beta+1)$,

(ii) each particle at its death gives birth to 0 or 2 particles with probabilities $1 /(\beta+1)$ and $\beta /(\beta+1)$,

(iii) if there are two particles born as a result of a death at $x$ then one is placed at $x$ and the other at a random location $y$ with distribution $\beta^{-1} b(x, y)$,

(iv) all the random variables and events in (i), (ii) and (iii) are independent.

It is easy to check that the description given above defines a unique process when the initial configuration is finite (see Athreya and Ney (1972), p. 105) and that this process makes transitions at the rates indicated above. From this construction it is apparent that starting from a finite initial configuration $\left\{x_{1}, \ldots, x_{n}\right\}$ the process has the same distribution as a superposition of $n$ independent processes which begin with one point at $x_{i}$.

Intuitively this should also be true for initial configurations which are infinite. To guarantee this we shall define the infinite particle system by the following construction. Let $\left\{\left(\eta_{t}^{x}, t \geqq 0\right), x \in R^{d}\right\}$ be a collection of independent copies of the process with $\eta_{0}^{x}=\{x\}$. To define the process starting from an initial configuration $\xi$ we let $\eta_{t}^{\xi}=\bigcup_{x \in \xi} \eta_{t}^{x}$.

The construction above shows that the process is additive in the sense of Harris (1978). Our process is a special type of additive process-it has independent components. The last property is crucial for our analysis. Our approach will be to first determine the behavior of the individual $\eta_{t}^{x}$ and then consider the infinite particle system as a superposition of the $\eta_{t}^{x}$. The results we have obtained are summarized below.

To describe these results we have to introduce some notation. Let $P^{x}$ and $P^{\xi}$ be the probability laws of the process $\eta_{t}^{x}$ and $\eta_{t}^{\xi}$ and let $E^{x}$ and $E^{\xi}$ be the corresponding expectations. If $\Lambda$ is a probability distribution on the space of configurations (this is described in Section 2 ) then we define a probability law $P^{\Lambda}$ and an expectation $E^{\Lambda}$ by $P^{\Lambda}(A)=\int \Lambda(d \xi) P^{\xi}(A)$. This is the probability law of the process starting from an initial distribution $\Lambda$.

The first step in analyzing the infinite particle system is to determine the mean number of particles in a compact set. In Section 3 we compute that

$$
E^{x} \eta_{t}(K)=e^{-t} \sum_{n=0}^{\infty} \frac{t^{n}}{n !} b_{n}(x, K)
$$

where $b_{n}(x, K)=\int d y b(x, y) b_{n-1}(y, K)$ and $b_{0}(x, K)=1_{K}(x)$. With this result it is easy to determine the behavior of the process when $\beta<1$. If $\xi$ is an initial 
configuration with

$$
\sup _{n} \int \xi(d x) \beta^{-n} b_{n}(x, A)=C<\infty
$$

i.e. if the density of particles is bounded in an appropriate sense, then $E^{\xi} \eta_{t}(A) \leqq C e^{t(\beta-1)}$. If $\beta<1$ this implies $\eta_{t}(A)$ converges to 0 in probability. The same conclusion holds for all compact sets $K$ if $b(x, y)=b(0, y-x)$ and the initial distribution is a translation-invariant point process with finite intensity.

The results given above show that the process 'dies out' when $\beta<1$. In the case $\beta>1$ we have the opposite behavior. If we let $\left|\eta_{t}\right|=\eta_{t}\left(R^{d}\right)$ then for each $x \in R^{d}\left|\eta_{t}^{x}\right|$ is a Markov branching process which evolves according to a probability law which is independent of $x$. It is known (see Athreya and Ney (1972), p. 111, p. 106) that as $t \rightarrow \infty e^{-(\beta-1) t}\left|\eta_{t}^{x}\right|$ converges almost surely to a limit $W_{x}$ which has $P\left\{W_{x}>0\right\}=P\left\{\eta_{t}^{x} \neq \varnothing\right.$ for all $\left.t\right\}=1-\left(1 \wedge \beta^{-1}\right)$ (see Athreya and Ney (1972), p. 112, p. 7).

This suggests that if the initial configuration $\xi$ has enough particles then for all open sets $G \eta_{t}(G) \rightarrow \infty$ in $P^{k}$ probability. To prove results of this type we need to know the particles are distributed in space at time $t$. To obtain this information we shall suppose that the process is spatially homogeneous, that is, there is a function $b$ defined on $R^{d}$ such that $b(x, y)=b(y-x)$. In this case the function $\beta^{-1} b(x, y)$ and its iterates are transition probabilities for a random walk and the processes $\left\{\eta_{t}^{x}, t \geqq 0\right\}$ are supercritical branching random walks.

These processes have been studied in various settings by Ney (1965), Kharmalov (1968), Joffe and Moncayo (1973), Asmussen and Kaplan (1976), Kaplan and Asmussen (1976), and others. Unfortunately none of their results supply the information we need to study the infinite particle system so in Section 4 we prove the following local limit theorem for Markovian supercritical random walks.

Theorem 4.1. Suppose $\int|y|^{2} b(y) d y<\infty$ and $\int y b(y) d y=0$. If $h>0$ and $x_{t} / t^{1 / 2} \rightarrow x$ then as $t \rightarrow \infty$

$$
(2 \beta t)^{d / 2} e^{-(\beta-1) t} \eta_{t}^{0}\left(x_{t}+[0, h]^{d}\right) \rightarrow h^{d} \varphi(x) W_{0}
$$

in $P^{0}$ probability. Here $\varphi$ is a d-dimensional normal density with mean 0 and covariance matrix $\sum_{i j}=(2 \beta)^{-1} \int y_{i} y_{j} b(y) d y$ and $W_{0}$ is the almost sure limit of $e^{-(\beta-1) t}\left|\eta_{t}^{0}\right|$.

The reader should note that the limit result in Theorem 4.1 is the one we should get if the particle locations were independent. That is, if $\tilde{\eta}_{t}^{x}$ is the point process which is constructed by assigning the particles alive at time $t$ independent locations with distribution $e^{-t(\beta-1)} E \eta_{t}^{x}(\cdot)$ then (1.2) holds with $\eta$ replaced by $\tilde{\boldsymbol{\eta}}$. 
In Section 5 we use Theorem 4.1 to give conditions on the initial configuration which guarantee that starting from $\eta, \eta_{t}(G)$ converges to $\infty$ in probability for all open sets $G$. The main result is the following.

Theorem 5.1. Suppose $\int|y|^{2} b(y) d y<\infty$ and let $\nu=(2 \beta)^{-1} \int y b(y) d y$. If for some $C<\infty$

$$
\lim _{t \rightarrow \infty} \eta\left([-C, C]^{d} t^{1 / 2}-\nu t\right)=\infty
$$

then for all open sets $G t^{d / 2} e^{-(\beta-1) t} \eta_{t}(G)$ converges to $\infty$ in $P^{n}$ probability.

In the special case $\nu=0$ the condition above becomes $|\eta|=\infty$ an obvious necessary condition for $\eta_{t}(G) \rightarrow \infty$ in probability. Another consequence of this result is the following theorem.

Theorem 5.2. If $\Lambda$ is a translation-invariant distribution with $\Lambda(\{\eta: \eta \neq \varnothing\})=$ 1 then for all $\theta<\beta-1$ and all open sets $G e^{-\theta t} \eta_{t}(G)$ converges to $\infty$ in $P^{\Lambda}$ probability.

For this result we do not need the assumption $\int|y|^{2} b(y) d y<\infty$ but having omitted this assumption we must weaken the conclusion.

In Sections 6,7 and 8 we consider the case $\beta=1$, again under the assumption that $b(x, y)=b(0, y-x)$. Under this assumption if the initial distribution is a translation-invariant point process with intensity $\lambda$ then the distribution of the process at any time $t$ is translation invariant and has intensity $\lambda e^{(\beta-1) t}$. This suggests that in the case $\beta=1$ (and only in this case) it is possible to have a non-trivial translation-invariant stationary distribution.

In Section 6 we show that if the random walk which takes steps according to the density $b^{0}(y)=\frac{1}{2}[b(y)+b(-y)]$ is transient then there is a non-trivial stationary distribution which is translation invariant. Using results of Debes, Kerstan, Liemant and Matthes (1970) we can then identify all the translationinvariant stationary distributions with finite intensity, and give conditions on the initial distribution $\Lambda$ which guarantee that starting from $\Lambda \eta_{t}$ converges in distribution as $t \rightarrow \infty$. The condition which is sufficient for convergence is satisfied by all translation-invariant point processes with finite intensity.

In Sections 7 and 8 we discuss what happens when the random walk generated by the density $b^{0}$ is recurrent. It is natural to conjecture that in this case there are no non-trivial translation-invariant stationary distributions with finite intensity, but $I$ have only been able to prove this for one-dimensional processes with $\int|y| b(y) d y<\infty$ and for two-dimensional processes with $\int|y|^{2} b(y) d y<\infty \dagger$. The proofs of these results are based on a simple necessary and sufficient condition for the existence of translation-invariant stationary

$\dagger$ After this paper was prepared the author learned that this conjecture has been proved by Kallenberg (1977) using different methods. 
distributions which is due to Liemant. To check that this condition is not satisfied we use the weak law of large numbers when $d=1$ and the local central limit theorem when $d=2$.

The conclusions in the last three sections are related to those obtained by Sawyer (1976) and Fleischman (1978) for processes which they call branching random fields. In these models particles move according to Brownian motion, live for an exponential amount of time and then die giving birth to $n$ offspring with probability $p_{n}$. In their formulation they allow the offspring to be introduced into the state according to a distribution $q_{n}\left(x, d y_{1}, \cdots, d y_{n}\right)$ on $\left(R^{d}\right)^{n}$ when the particle dies at $x$ but for most of their conclusions they require all the offspring to be born at the point where the parent died. Under this assumption they proved that the conclusions of Theorems 6.1 and 8.5 hold for branching random fields (see Sawyer (1976), Theorem 4.2 and Fleischman (1978), Theorem 4.1). Their techniques of proof are different however because they can rely on the explicit formulas available for branching Brownian motions.

It is likely that the results we have stated above can be extended to the general branching random fields defined by Sawyer. The results should be the same in the subcritical and supercritical cases but in the critical case the conclusions of Sections 7 and 8 will require the additional assumption that $p_{1} \neq 1$. The case $p_{1}=1$ corresponds to particles undergoing independent motions. In this case it is known that the $d$-dimensional Poisson process is invariant in any $d \geqq 1$ (see Doob (1953)) and that starting from a translationinvariant distribution $\Lambda$ with finite intensity, $\eta_{t}$ converges in distribution to a mixture of Poisson processes (see Stone (1968), Matthes (1972)).

\section{Preliminaries}

In this section we shall give some definitions and prove some preliminary results which will be needed in Sections 3-8. The first of these results concerns the description of the process. The construction described in the introduction gives us (for almost every $\omega$ ) a mapping from $[0, \infty)$ to the countable subsets of $R^{d}$. To be able to discuss the distribution of the process at time $t$ we need to introduce a suitable $\sigma$-algebra on the state space. To do this we need some definitions:

Let $M$ be the set of all measures $\mu$ on $R^{d}$ which have $\mu(A) \in\{0,1, \cdots, \infty\}$ for all Borel sets $A$.

We endow $M$ with the topology of weak convergence. That is $\mu_{n} \rightarrow \mu$ in $M$ if and only if $\lim _{\inf _{n \rightarrow \infty}} \mu_{n}(G) \geqq \mu(G)$ for all open sets $G \subseteq R^{d}$.

Let $\mu$ be the Borel subsets of $M$. $\mu$ is also the smallest $\sigma$-algebra such that for each Borel set $A$ the mapping $\mu \rightarrow \mu(A)$ is $\mu$-measurable. 
Let $L$ be the collection of countable subsets of $R^{d}$. Let $\pi: L \rightarrow M$ be the mapping which has $(\pi l)(A)=|A \cap l|$. Let $\mathscr{L}=\left\{\pi^{-1} A: A \in \mathcal{M}\right\}$.

It is easy to see that if $l_{1}, l_{2} \in L$ and $l_{1} \neq l_{2}$ then $\pi l_{1} \neq \pi l_{2}$ (consider $l_{2}-l_{1}, l_{1}-$ $\left.l_{2} \subset R^{d}\right)$. From this it follows that the measure spaces $(L, \mathscr{L})$ and $(M, \mathcal{M})$ are isomorphic, and so we may define the distribution of $\eta_{t}$ as a probability measure on either space. Following the traditional practice we will define the probability measure on $(M, M)$. In what follows we shall use the terminology which has become associated with probability measures on $(M, M)$ (see Daley and Vere-Jones (1972)). The reader should note that although we shall call the distributions point processes we do not require a priori that the probability measure be concentrated on $M^{\prime}=\{\mu \in M: \mu(K)<\infty$ for all compact sets $K\}$.

Having defined the distributions of the process the next notion which we need to introduce is the transition semigroup: if $\Lambda$ is a distribution on $(M, M)$ let $\Lambda T_{t}$ be the distribution on $(M, \mathcal{M})$ which has

$$
\Lambda T_{t}(\pi A)=\int_{M} \Lambda(d \xi) P\left(\eta_{t}^{\xi} \in A\right) \text { for all } A \in \mathscr{L} .
$$

If the distribution of $\eta_{s}$ is $\Lambda$ then $\Lambda T_{t}$ is the distribution of $\eta_{s+t}$.

For some of the results of Sections 6 and 7 we need to know that the semigroup $T_{t}$ has the Feller property. To show this we observe that $\eta_{t}^{\mathrm{x}} \stackrel{d}{=} x+\eta_{t}^{0}$ so if $\xi_{n}$ is a sequence of elements of $M$ which converge to $\xi$ then we can construct a sequence of random variables $\tilde{\eta}_{t}^{n}, n \geqq 1$ on a probability space in such a way that $\tilde{\eta}_{t}^{n} \stackrel{d}{=} \eta_{t}^{\xi_{n}}$ and $\tilde{\eta}_{t}^{n}$ converges almost surely to a limit $\tilde{\eta}_{t}^{\infty}$ which has the same distribution as $\eta_{1}^{\xi}$. This shows that if $G$ is an open subset of $M$, $\xi \rightarrow P\left(\eta_{t}^{\xi} \in G\right)$ is a lower semicontinuous function.

From this it follows easily that if $\Lambda_{n}$ converges weakly to $\Lambda$ (as a sequence of probability measures on $M$ ) then

$$
\liminf _{t \rightarrow \infty} \Lambda_{n} T_{t}(G) \geqq \Lambda T_{t}(G) .
$$

This shows the mapping $\Lambda \rightarrow \Lambda T_{t}$ is continuous and hence that $T_{t}$ has the Feller property.

It is easy to show that the transition semigroup has other desirable properties-e.g. for 'nice' $\Lambda, t \rightarrow \Lambda T_{t}$ is continuous-but we shall not pursue this here. The rest of this section is devoted to proving a result about a special class of distributions.

We begin by stating two definitions. A probability measure $\Lambda$ on $(L, \mathscr{L})$ is translation invariant if for all Borel sets $A \subset L \Lambda(A)=\Lambda(x+A)$. Here $x+A=$ $\{x+\xi: \xi \in A\} \subset L$ and $x+\xi=\{x+y: y \in \xi\} \subset R^{d}$. A probability measure $\Lambda$ on $(M, \mu)$ is said to be translation invariant if the measure $\Lambda \circ \pi$ is.

In words, a distribution $\Lambda$ is translation invariant if its probability law is 
spatially homogeneous. The mean measure $m(A)=\int \Lambda(d \eta) \eta(A)$ of a translation-invariant distribution is translation invariant and hence a constant times Lebesgue measure. This constant is called the intensity of the point process.

Theorem 2.1. If $\Lambda$ is a translation-invariant point process with finite intensity then there is a function $\gamma$ defined on $M$ which has the following property:

$$
\int \Lambda(d \eta)\left|\frac{\eta\left(\left\{x: 0 \leqq x \leqq n^{k}\right\}\right)}{n_{1}^{k} n_{2}^{k} \cdots n_{d}^{k}}-\gamma(\eta)\right| \rightarrow 0
$$

whenever $n^{k}$ is a sequence of points in $Z^{d}$ which have $n_{i}^{k} \rightarrow \infty$ for all $1 \leqq i \leqq d$. Furthermore $\Lambda(\{\eta: \gamma(\eta)>0\})=\Lambda(\{\eta: \eta \neq \varnothing\})$.

Remark. In what follows we shall refer to $\gamma$ as the asymptotic density for the point process.

Proof. The first result is an immediate consequence of the following multiparameter ergodic theorem which is due to Dunford (1951):

Let $N=\{1,2, \cdots\}$, let $\left\{X_{j}, j \in N^{d}\right\}$ be an array of random variables and define the shift $\theta_{i}$ by

$$
X_{j_{1}, \cdots, j_{i}, \cdots, j_{d}}\left(\theta_{i} \omega\right)=X_{j_{1}, \cdots, j_{i}+1, \cdots, j_{d}}(\omega) .
$$

Suppose that $X_{1}, \cdots, 1$ is integrable and that for each $1 \leqq i \leqq d\left\{X_{i}, j \in N^{d}\right\}$ and $\left\{X_{i} \circ \theta_{i}, j \in N^{d}\right\}$ have the same distribution.

If $n^{k}$ is a sequence of elements of $N^{d}$ with $n_{i}^{k} \rightarrow \infty$ for $1 \leqq i \leqq d$ then $S_{n^{k}} /\left(n_{1}^{k} n_{2}^{k} \cdots n_{d}^{k}\right)$ converges in $L^{1}$.

(This statement of the result can be found in Smythe (1976) where an extension to multiparameter subadditive processes is proved.)

To prove the second result we observe that from the ergodic theorem $\gamma$ is invariant under each shift so

$$
\begin{aligned}
E^{\Lambda}\left[\eta\left([0,1]^{d}\right) ; \gamma(\eta)>0\right] & =E^{\Lambda}\left[n^{-d} \eta\left([0, n]^{d}\right) ; \gamma(\eta)>0\right] \\
& \geqq E^{\Lambda}[\gamma(\eta) ; \gamma(\eta)>0]-\int \Lambda(d \eta)\left|n^{-d} \eta\left([0, n]^{d}\right)-\gamma(\eta)\right| .
\end{aligned}
$$

Letting $n \rightarrow \infty$ gives

$$
E^{\Lambda}\left[\eta\left([0,1]^{d}\right) ; \gamma(\eta)>0\right]=\int \Lambda(d \eta) \gamma(\eta)=E^{\Lambda}\left[\eta\left([0,1]^{d}\right)\right] .
$$

Since $\Lambda$ is translation invariant this shows $\Lambda(\{\eta: \gamma(\eta)=0, \eta \neq \varnothing\})=0$.

3. $\beta<1$

In this section we shall assume $\beta<1$ and obtain conditions which imply that 
starting from an initial configuration $\xi, \eta_{t}(K)$ converges to 0 in probability for each compact $K \subset S$.

If there is initially one particle present $Z(t)=\left|\eta_{t}^{x}\right|$ is a Markov branching process. In this case $E\left|\eta_{t}^{x}\right|=e^{t(\beta-1)}$ (see Athreya and Ney (1972), (4), p. 108) so $P\left\{\left|\eta_{t}^{x}\right| \geqq 1\right\} \rightarrow 0$ as $t \rightarrow \infty$. To obtain the corresponding results for initial configurations which are infinite we need to know how the particles are distributed in space. To do this we need some notation. Let

$$
\begin{aligned}
& m_{1}(x, K)=\frac{1}{2} 1_{K}(x)+\frac{1}{2} \int_{K} \beta^{-1} b(x, y) d y \\
& \text { and } \quad m_{n+1}(x, K)=\int m_{n}(x, d y) m(y, K) \text { for } n \geqq 1 .
\end{aligned}
$$

$m_{n}(x, K)$ is the fraction of the $n$th generation that will be born in $K$ when the process starts with one particle at $x$. To compute $E^{x} \eta_{t}(K)$ we observe that $e^{-t(\beta+1)} t^{n}(\beta+1)^{n} / n$ ! is the probability that there are exactly $n$ arrivals before $t$ in a Poisson process with rate $\beta+1$, and $(2 \beta / \beta+1)^{n}$ is the expected size of the $n$th generation so

$$
E \eta_{t}^{x}(K)=\sum_{n=0}^{\infty} e^{-t(\beta+1)} \frac{t^{n}}{n !}(2 \beta)^{n} m_{n}(x, K) .
$$

For a more detailed derivation of this result see Ney (1965).

For some of our later results it is convenient to express $E \eta_{t}^{x}(K)$ directly in terms of $b$. To do this we let

$$
\begin{gathered}
b_{0}(x, K)=1_{K}(x) \\
\text { and } b_{n}(x, K)=\int b(x, y) b_{n-1}(y, A) d y \text { for } n \geqq 1
\end{gathered}
$$

and note that from (3.1) if $p_{n}(x, K)=\beta^{-n} b_{n}(x, K)$ then

$$
m_{n}(x, K)=2^{-n} \sum_{j=0}^{n}\left(\begin{array}{l}
n \\
j
\end{array}\right) p_{j}(x, K)
$$

Substituting this into (3.2) gives

$$
\begin{aligned}
E \eta_{t}^{x}(K) & =e^{-t(\beta+1)} \sum_{n=0}^{\infty} \frac{(t \beta)^{n}}{n !} \sum_{j=0}^{n}\left(\begin{array}{l}
n \\
j
\end{array}\right) p_{j}(x, K) \\
& =e^{-t} \sum_{j=0}^{\infty} \frac{(t \beta)^{j}}{j !} p_{j}(x, K) .
\end{aligned}
$$

From this formula we can obtain conditions which guarantee $P^{\xi}\left\{\eta_{t}(K)<\infty\right\}=$ 1 for $\beta<\infty$ and that $P^{\xi}\left\{\eta_{t}(K)=0\right\} \rightarrow 1$ for $\beta<1$. These conclusions are both obtained from the observation that if the initial configuration is $\xi$ and $A$ is a 
Borel subset of $R^{d}$ then

$$
\begin{aligned}
E \eta_{t}^{\xi}(A) & =E \int \xi(d x) \eta_{t}^{x}(A) \\
& =e^{-t} \sum_{n=0}^{\infty} \frac{(t \beta)^{n}}{n !} \int \xi(d x) p_{n}(x, A) .
\end{aligned}
$$

From this equality we see that if the set $A$ has $\sup _{n} \int \xi(d x) p_{n}(x, A)=C<\infty$ then $E \eta_{t}^{\xi}(A) \leqq C e^{t(\beta-1)}$. From this it follows that if $\beta<\infty, \eta_{t}(A)<\infty P^{\xi}$ almost surely and if $\beta<1 P^{\xi}\left\{\eta_{t}(A) \geqq 1\right\} \rightarrow 0$.

A similar argument, which we shall now give, shows that if $b(x, y)=b(y-x)$ then the same conclusion holds for all compact sets $K$ when the initial distribution $\Lambda$ is translation invariant and has finite intensity $\lambda$. In this case it follows from (3.5) that

$$
E^{\Lambda} \eta_{t}(K)=e^{-t} \sum_{n=0}^{\infty} \frac{(t \beta)^{n}}{n !} \int \Lambda(d \xi) \int \xi(d x) p_{n}(x, K)=e^{t(\beta-1)} \lambda|K|
$$

and hence that $\eta_{t}(K)<\infty P^{\Lambda}$ almost surely if $\beta<\infty$, and $P^{\Lambda}\left\{\eta_{t}(K) \geqq 1\right\} \rightarrow 0$.

By using the inequality $E^{\Lambda} \eta_{t}(K) \leqq C e^{t(\beta-1)}$ in a slightly different manner we can improve the results stated above in the case $\beta<1$. To do this we observe

$$
E^{\Lambda} \int_{0}^{\infty} \eta_{t}(K) d t=\int_{0}^{\infty} E^{\Lambda} \eta_{t}(K) d t \leqq C \int_{0}^{\infty} e^{t(\beta-1)} d t<\infty .
$$

Since the lifetimes are independent of the configuration this implies that with $P^{\Lambda}$ probability $1, \eta_{t}(K)=0$ for $t \geqq t_{0}(\omega)$. It follows from this that for $\Lambda$ almost every $\xi$ the same statement has $P^{\xi}$ probability 1 .

\section{Supercritical branching random walks}

In this section we shall assume $\beta>1$, and obtain a limit law for the number of particles in an open set $G$ at time $t$ when the system is initiated by one particle located at zero. In other words we shall prove a local limit theorem for a Markovian supercritical branching random walk. The proof of this result is based on the one given in Kaplan and Asmussen (1976). With some effort the proof given below can be extended to show the same conclusion holds for age-dependent branching processes.

Theorem 4.1. Suppose $\int|y|^{2} b(y) d y<\infty$ and $\int y b(y) d y=0$. If $h>0$ and $x_{t} / t^{1 / 2} \rightarrow x \in R^{d}$ then

$$
(2 \beta t)^{d / 2} e^{-(\beta-1) t} \eta_{t}^{0}\left(x_{t}+(0, h)^{d}\right) \rightarrow h^{d} \varphi(x) W
$$

in probability. Here $\varphi$ is the density of a normal random vector with mean 0 and 
covariance matrix $\sum_{i j}=(2 \beta)^{-1} \int y_{i} y_{j} b(y) d y$ and $W$ is the almost sure limit of $e^{-(\beta-1) t}\left|\eta_{t}^{0}\right|$.

Remark. The rest of the section is devoted to the proof of this result and may be omitted without loss of continuity.

Proof. Let $K_{t}=x_{t}+(0, h)^{d}$. Let $s<t$ and let $\eta_{s, t}(y, K)$ be the number of particles in $K$ at time $t$ with an ancestor at $y$ at time $s$. With this definition we can write

$$
\eta_{t}\left(K_{t}\right)=\sum_{y \in \eta_{s}} \eta_{s, t}\left(y, K_{t}\right)
$$

Now the random variables $\eta_{s, t}\left(y, K_{t}\right)$ are independent, so letting $m(t, x, K)=$ $E^{x} \eta_{t}(K)$ we can write

$$
(2 \beta t)^{d / 2} e^{-(\beta-1) t} \eta_{t}\left(K_{t}\right)-h^{d} \Phi(x) W=A_{s, t}+B_{s, t}+C_{s}
$$

where

$$
\begin{aligned}
A_{s, t} & =(2 \beta t)^{d / 2} e^{-(\beta-1) t} \sum_{y \in \eta_{s}}\left[\eta_{s, t}\left(y, K_{t}\right)-m\left(t-s, y, K_{t}\right)\right], \\
B_{s, t} & =e^{-(\beta-1) s} \sum_{y \in \eta_{s}}\left[(2 \beta t)^{d / 2} e^{-(\beta-1)(t-s)} m\left(t-s, y, K_{t}\right)-h^{d} \Phi(x)\right], \\
C_{s} & =h^{d} \Phi(x)\left(e^{-(\beta-1) s}\left|\eta_{s}\right|-W\right) .
\end{aligned}
$$

To prove Theorem 4.1 we shall show that if $t \rightarrow \infty$ and $s(t)=t^{\rho}$ with $0<\rho<1$ then $A_{s(t), t}, B_{s(t), t}$ and $C_{s(t)}$ converge to zero in probability.

The last term is the easiest to deal with. Since $s(t)=t^{\rho}$ and $\rho>0, s(t) \rightarrow \infty$ as $t \rightarrow \infty$ and so $e^{-(\beta-1) s(t)} \eta_{s(t)}$ converges almost surely to $W$, the random variable described in the statement of the theorem. From this it is immediate that $C_{s(t)} \rightarrow 0$.

To estimate $A_{s, t}$ we observe that the random variables $\eta_{s, t}\left(y, K_{t}\right)$ are independent and, conditional on $y \in \eta_{s}, \eta_{s, t}\left(y, K_{t}\right)$ has the same distribution as $\eta_{t-s}^{y}\left(K_{t}\right)$. Now

$$
E \eta_{t-s}^{y}\left(K_{t}\right)=m\left(t-s, y, K_{t}\right)
$$

and from Formula (5) (Athreya and Ney (1972), p. 109) there is a constant $C<\infty$ such that

$$
E\left[\eta_{t-s}^{y}\left(K_{t}\right)\right]^{2} \leqq E\left|\eta_{t-s}^{y}\right|^{2} \leqq C e^{2(\beta-1)(t-s)}
$$

so it follows from the central limit theorem that $A_{s(t), t} \rightarrow 0$ in probability whenever $t^{d / 2} e^{-(\beta-1) s(t) / 2} \rightarrow 0$. This condition holds if $\beta>1$ and $s(t)=t^{\rho}$ with $\rho>0$.

To show $B_{s(t), t} \rightarrow 0$ we shall need an estimate for $Z_{n}(t)$, the number of particles alive at time $t$ who are members of the $n$th generation. For this we 
shall use the following result which is due to Samuels (1971) (see relation (16), p. 665).

Lemma 4.2. If $\left(a_{t}-2 \beta t\right) / t^{1 / 2} \rightarrow-\infty$ and $\left(b_{t}-2 \beta t\right) / t^{1 / 2} \rightarrow \infty$ then

where

$$
E\left(e^{-(\beta-1) t} \sum_{n=a_{1}}^{b_{t}} Z_{n}(t)-e^{-(\beta-1) t} Z(t)\right)^{2} \rightarrow 0
$$

$$
Z(t)=\sum_{n=0}^{\infty} Z_{n}(t)=\left|\eta_{t}^{0}\right|
$$

In words most of the population at time $t$ are from generations $n$ in the interval $\left[a_{t}, b_{t}\right]$.

Lemma 4.3. If $y_{t} / t^{1 / 2} \rightarrow 0$ and $K_{t}^{\prime}=x_{t}^{\prime}+(0, h)^{d}$ with $x_{t}^{\prime} / t^{1 / 2} \rightarrow x$ then

$$
(2 \beta t)^{d / 2} e^{-(\beta-1) t} m\left(t, y_{t}, K_{t}^{\prime}\right) \rightarrow h^{d} \varphi(x)
$$

where $\varphi$ is a normal density with mean 0 and covariance $\Sigma$.

Proof. Let $a_{t} \leqq 2 \beta t \leqq b_{t}$ be such that $\left(b_{t}-a_{t}\right) / t \rightarrow 0,\left(a_{t}-2 \beta t\right) / t^{1 / 2} \rightarrow-\infty$ and $\left(b_{t}-2 \beta t\right) / t^{1 / 2} \rightarrow \infty$.

$$
\begin{aligned}
(2 \beta t)^{d / 2} e^{-(\beta-1) t} m\left(t, y_{t}, K_{t}^{\prime}\right) & =(2 \beta t)^{d / 2} e^{-(\beta-1) t} \sum_{n=0}^{\infty} m_{n}\left(y_{t}, K_{t}^{\prime}\right) E Z_{n}(t) \\
& \geqq e^{-(\beta-1) t} \sum_{n=a_{t}}^{b_{t}}(2 \beta t)^{d / 2} m_{n}\left(y_{t}, K_{t}^{\prime}\right) E Z_{n}(t) .
\end{aligned}
$$

Since $a_{t} / t$ and $b_{t} / t$ converge to $2 \beta$, and $m_{n}(0, \cdot)$ is the distribution of the sum of $n$ independent random variables with distribution $m_{1}(0, \cdot)$ it follows from the local central limit theorem (see Stone (1965), Theorem 1) that the above

$$
\sim \varphi(x) h^{d} e^{-(\beta-1) t} \sum_{n=a_{t}}^{b_{t}} E Z_{n}(t)
$$

Applying Lemma 4.2 now gives the above

$$
\sim \varphi(x) h^{d} e^{-(\beta-1) t} E Z(t)=\varphi(x) h^{d} .
$$

To complete the proof we have to show that

$$
e^{-(\beta-1) t} \sum_{n \notin\left[a_{t}, b_{t}\right]}(2 \beta t)^{d / 2} m_{n}\left(y_{t}, K_{t}^{\prime}\right) E Z_{n}(t) \rightarrow 0 .
$$

The sum over $n>b_{t}$ is easy to estimate. Since $b_{t} \geqq 2 \beta t$

$$
e^{-(\beta-1) t} \sum_{n>b_{t}}(2 \beta t)^{d / 2} m_{n}\left(y_{t}, K_{t}^{\prime}\right) E Z_{n}(t) \leqq e^{-(\beta-1) t} \sum_{n>b_{t}} n^{d / 2} m_{n}\left(y_{t}, K_{t}^{\prime}\right) E Z_{n}(t) .
$$


From the local central limit theorem there is a $C<\infty$ such that

$$
\sup _{n, z} n^{d / 2} m_{n}\left(z,(0, h)^{d}\right) \leqq C .
$$

From this it follows that the sum over $n>b_{t}$ is

$$
\leqq C e^{-(\beta-1) t} \sum_{n>b_{t}} E Z_{n}(t)
$$

which converges to 0 by Lemma 4.2.

The sum over $n<a_{t}$ requires more care. Let $\delta<(\beta-1) / 2 \log (2 \beta / \beta+1)$

$$
e^{-(\beta-1) t} \sum_{n \leqq \delta t}(2 \beta t)^{d / 2} m_{n}\left(y_{t}, K_{t}^{\prime}\right) E Z_{n}(t) \leqq\left((2 \beta t)^{d / 2} e^{-(\beta-1) t / 2}\right) e^{-(\beta-1) t / 2} \sum_{n \leqq \delta t} E Z_{n}(t) .
$$

Now

$$
\begin{aligned}
\sum_{n \leqq \delta t} E Z_{n}(t) \leqq \sum_{n \leqq \delta t}\left(\frac{2 \beta}{\beta+1}\right)^{n} & =\left(\frac{2 \beta}{\beta+1}\right)^{[\delta t]}\left(\sum_{n=0}^{[\delta t]}\left(\frac{\beta+1}{2 \beta}\right)^{n}\right) \\
& \leqq\left(\frac{2 \beta}{\beta+1}\right)^{\delta t}\left[1-\frac{\beta+1}{2 \beta}\right]^{-1}=\frac{2 \beta}{\beta-1} \exp \left(t \delta \log \left(\frac{2 \beta}{\beta+1}\right)\right) .
\end{aligned}
$$

Since $\delta<(\beta-1) /(2 \log (2 \beta /(\beta+1)))$ this shows that

$$
e^{-(\beta-1) t / 2} \sum_{n \leqq \delta t} E Z_{n}(t) \rightarrow 0
$$

and hence that the sum over $n \leqq \delta t$ converges to 0 .

It is now routine to show the last remaining piece converges to 0 .

$$
\begin{aligned}
e^{-(\beta-1) t} \sum_{\delta t \leq n \leqq a_{t}}(2 \beta t)^{d / 2} m_{n}\left(y_{t}, K_{t}^{\prime}\right) E Z_{n}(t) & \\
& \leqq\left(\frac{2 \beta}{\delta}\right)^{d / 2} e^{-(\beta-1) t} \sum_{\delta t \leq n \leqq a_{t}} n^{d / 2} m_{n}\left(y_{t}, K_{t}^{\prime}\right) E Z_{n}(t)
\end{aligned}
$$

which converges to 0 by the same argument used for the sum over $n>b_{t}$.

The proof of Lemma 4.3 is now complete. To finish the proof of Theorem 4.1 it remains to show the following

Lemma 4.4. If $s(t)=t^{\rho}$ with $\rho<1$ then $B_{s(t), t} \rightarrow 0$ in probability.

Proof. Pick $\varepsilon_{t} \rightarrow 0$ so that $(s(t) / t)^{1 / 2} / \varepsilon_{t} \rightarrow 0$. From the local central limit theorem there is a constant $C<\infty$ such that

$$
\left|(2 \beta t)^{d / 2} e^{-(\beta-1) t} m\left(t, y,(0, h)^{d}\right)\right| \leqq C
$$

for all $t \geqq 0, y \in R^{d}$. From this it follows that

$$
\begin{aligned}
B_{s, t} \leqq e^{-(\beta-1) s} \int_{|y|<t^{1 / 2} \varepsilon_{t}} \eta_{s}^{0}(d y)\left[(2 \beta t)^{d / 2} e^{-(\beta-1)(t-s)} m\left(t-s, y, K_{t}\right)-\varphi(x) h^{d}\right] \\
+e^{-(\beta-1) s} \eta_{s}^{0}\left(\left\{y:|y| \geqq t^{1 / 2} \varepsilon_{t}\right\}\right) C(t / t-s)^{d / 2} .
\end{aligned}
$$


Since $e^{-(\beta-1) s} \eta_{s}^{0}$ converges in probability to $W<\infty$ it follows from Lemma 4.3 that the first term converges to 0 . To estimate the second term we observe that $e^{-(\beta-1) s} E Z(s)=1$ so

$$
\begin{aligned}
e^{-(\beta-1) s} E \eta_{s}^{0}\left(\left\{y:|y|>t^{1 / 2} \varepsilon_{t}\right\}\right) \leqq & \left(e^{-(\beta-1) s} \sum_{n>(2 \beta+1) s} E Z_{n}(s)\right) \\
& +\sup _{n \leqq(2 \beta+1) s} m_{n}\left(0,\left\{y:|y|>t^{1 / 2} \varepsilon_{t}\right\}\right) .
\end{aligned}
$$

From Lemma 4.2 the first term converges to 0 . To estimate the second we observe that from the central limit theorem, if we let

$$
\sup _{n} m_{n}\left(0,\left\{y:|y|>x n^{1 / 2}\right\}\right)=c(x)
$$

then $c(x) \leqq 1$ and $c(x) \downarrow 0$ as $x \uparrow \infty$. From this we get

$$
\sup _{n \leqq(2 \beta+1) s} m_{n}\left(0,\left\{y:|y|>t^{1 / 2} \varepsilon_{t}\right\}\right) \leqq c\left(\varepsilon_{t}(t / s(2 \beta+1))^{1 / 2}\right)
$$

so if $s=t^{\rho}, \rho<1$ it follows from the choice of $\varepsilon_{t}$ that the right side converges to 0 as $t \rightarrow \infty$. This completes the proof of Lemma 4.4 and Theorem 4.1.

\section{5. $\beta>1$}

In this section we shall assume $\beta>1$ and obtain limit theorems for the number of particles in an open set $G \subset R^{d}$ when the process starts from an infinite initial configuration. The first result is an easy consequence of Theorem 4.1.

Theorem 5.1. Suppose $\int|y|^{2} b(y) d y<\infty$. If $\int y b(y) d y=0$ and $\xi$ is a configuration with $|\xi|=\infty$ then for all open sets $G, t^{d / 2} e^{-(\beta-1) t} \eta_{t}(G) \rightarrow \infty$ in $P^{\xi}$ probability. If $\int y b(y) d y=\beta \nu$ then the same conclusion holds if for some $C<\infty$

$$
\lim _{t \rightarrow \infty} \xi\left([-C, C]^{d} t^{1 / 2}-\nu t\right)=\infty .
$$

Proof. It suffices to prove the result for sets of the form $G=x+(0, h)^{d}$. If we let $\eta_{0, t}(y, G)$ be the number of points in $G$ at time $t$ with an ancestor at $y$ at time 0 then we have

$$
\eta_{t}(G)=\sum_{y \in \xi} \eta_{0, t}(y, G)
$$

and the $\eta_{0, t}(y, G)$ are independent random variables. Now if for each $y$ we define $W_{y}$ to be the almost sure limit of $e^{-(\beta-1) t} \eta_{0, t}(y, G)$ then from Theorem 4.1

$$
(2 \beta t)^{d / 2} e^{-(\beta-1) t} \eta_{0, t}(y, G) \rightarrow \varphi(0) h^{d} W_{y}
$$


Since the $W_{\mathrm{y}}$ are independent and identically distributed random variables with $P\left\{W_{y}>0\right\}>0$ it follows that $t^{d / 2} e^{-(\beta-1) t} \eta_{t}(G) \rightarrow \infty$.

The second conclusion is proved in a similar way. If we let $J_{t}=$ $[-C, C]^{d} t^{1 / 2}-\nu t$ then

$$
\eta_{t}(G) \geqq \sum_{y \in \xi \cap J_{t}} \eta_{0, t}(y, G) .
$$

From Theorem 4.1 (generalized to $\nu \neq 0$ ) we have that if $y_{t} \in J_{t}$ and $t \rightarrow \infty$

$$
\liminf _{t \rightarrow \infty} P\left((2 \beta t)^{d / 2} e^{-(\beta-1) t} \eta_{0, t}\left(y_{t}, G\right)\right) \geqq \frac{h^{d}}{2} W_{y_{t}}\left(\inf _{x \in[-c, c]^{d}} \varphi(x)\right)=1 .
$$

Since the random variables $W_{x}$ are independent and $P\left(W_{x}>0\right)>0$ it follows from this that if $\left|\xi \cap J_{t}\right| \rightarrow \infty$ then for all $M<\infty$

$$
P^{\xi}\left(t^{d / 2} e^{-(\beta-1) t} \eta_{t}(K) \leqq M\right) \rightarrow 0 .
$$

which completes the proof.

Theorem 5.1 can be generalized in two ways: (a) the restriction $\int|y|^{2} b(y) d y<\infty$ may be removed and (b) in the case $\nu \neq 0$ we can weaken the condition $\lim _{t \rightarrow \infty} \xi\left([-C, C]^{d} t^{1 / 2}-\nu t\right)=\infty$.

To extend Theorem 5.1 to the case $\int|y|^{2} b(y) d y=\infty$ we observe that if $\int b(y) d y>1$ then there is a bounded set $B$ such that $\int b(y) 1_{B}(y) d y>1$. Let $\bar{\eta}_{t}$ be the particle system associated with the birth rate $b(y) 1_{B}(y)$. Since $b(y) 1_{B}(y) \leqq b(y)$ it is easy to see that for each $x \bar{\eta}_{t}^{x}$ and $\eta_{t}^{x}$ can be constructed on the same probability space so that $\bar{\eta}_{t}^{x}(K) \leqq \eta_{t}^{x}(K)$ for all $t, K$. Once this is done if we take for each $x \in R^{d}$ an independent copy of the process $\left(\bar{\eta}_{t}^{x}, \eta_{t}^{x}\right)$ then we can obtain a pair of processes in which $\bar{\eta}_{t}^{\xi}(K) \leqq \eta_{t}^{\xi}(K)$ for all initial configurations $\xi$.

Using this observation we can obtain the following result.

Theorem 5.2. If $\Lambda$ is a translation-invariant distribution with $\Lambda(\{\eta: \eta \neq \oslash\})=$ 1 then for all $\theta<\beta-1$ and all open sets $G, e^{-\theta t} \eta_{t}(G)$ converges to $\infty$ in $P^{\Lambda}$ probability.

Proof. For each $\theta<\beta-1$ there is a compact set $K$ such that $\beta_{K}=$ $\int b(y) 1_{K}(y) d y>1+\theta$. If $\bar{\nu}=\beta_{K}^{-1} \int y b(y) 1_{K}(y) d y$ then from Theorem 2.1 and the fact that $\Lambda$ is translation invariant it follows that for all $y>0$

$$
\liminf _{t \rightarrow \infty} P^{\Lambda}\left\{\xi: \xi\left([-1,1]^{d} t^{1 / 2}-\bar{\nu} t\right) / t^{d / 2}>y\right\} \geqq P^{\Lambda}\left\{\eta: \gamma(\eta)>y / 2^{d}\right\}
$$

so $\xi\left([-1,1]^{d} t^{1 / 2}-\bar{\nu} t\right) \rightarrow \infty$ in $P^{\Lambda}$ probability. It now follows easily from the proof of the second conclusion of Theorem 5.1 that for all open sets $G, e^{-\theta t} \eta_{t}(G)$ converges to $\infty$ in $P^{\Lambda}$ probability. 
Theorem 5.2 gives a fairly complete description of what occurs when the system starts from an initial distribution which is translation invariant. For individual configurations or non-translation-invariant initial distributions it is difficult to state a definitive result. For a given function $b$ with $\int b(y) d y>1$ there are many sets $K$ with the property that $\int b(y) 1_{K}(y) d y>1$. Since $\int y b(y) 1_{K}(y) d y$ may take on different values for different sets $K$ this leads through Theorem 5.1 to a variety of sufficient conditions for $\eta_{t}(G) \rightarrow \infty$. Combining all the possibilities gives the following result.

Theorem 5.3. Let $\mathscr{V}=\left\{\int y b(y) 1_{K}(y) d y: \int b(y) 1_{K}(y) d y>1\right\}$. If $0 \in \mathscr{V}$ and $|\eta|=\infty$ or more generally, if for some $C<\infty, \bar{\nu} \in \mathcal{V}$

$$
\lim _{t \rightarrow \infty} \eta\left([-C, C]^{d} \sqrt{ } t-\bar{\nu} t\right)=\infty
$$

then $\eta_{t}(K) \rightarrow \infty$ in $P^{\eta}$ probability.

Remark. In the case $0 \in \mathcal{V}$ the condition $|\eta|=\infty$ is clearly also necessary. If $0 \notin \mathcal{V}$, however, the condition is probably far from the best possible. I have not been able to determine a general necessary condition for $\eta_{t}(G) \rightarrow \infty$ in $P^{\eta}$ probability.

\section{6. $\beta=1$, the transient case}

In this section we shall show that if $\beta=1$ and the random walk which has steps with distribution $b^{0}(y)=\frac{1}{2}[b(y)+b(-y)]$ is transient then there is a non-trivial stationary distribution. Once we have done this we can use results of Debes, Kerstan, Liemant and Matthes (1970) to (i) show that for every $\rho \geqq 0$ there is exactly one ergodic translation-invariant stationary distribution with intensity $\rho$ and (ii) give a sufficient condition for $\Lambda T_{t}$ to converge.

To prove the existence of a stationary distribution we consider what happens when the initial configuration is a Poisson process $\mathscr{X}$ with intensity 1 . In this case if $A_{1}$ and $A_{2}$ are open sets then

$$
E^{\mathscr{E}} \eta_{t}\left(A_{1}\right)=\int d x m\left(t, x, A_{1}\right)
$$

where $m\left(t, x, A_{1}\right)=e^{-t} \sum_{n=0}^{\infty}\left(t^{n} / n !\right) b_{n}\left(x, A_{1}\right)$ and

$$
E^{\mathscr{L}}\left[\eta_{t}\left(A_{1}\right) \eta_{t}\left(A_{2}\right)-\eta_{t}\left(A_{1} \cap A_{2}\right)\right]=w\left(t, A_{1}, A_{2}\right)
$$

where

$$
w\left(t, A_{1}, A_{2}\right)=\int d x \int_{0}^{t} d s \int m(t-s, x, d y) K_{2}\left(y, m\left(s, \cdot, A_{1}\right), m\left(s, \cdot, A_{2}\right)\right)
$$


and

$$
K_{2}(y, f, g)=\frac{1}{2}\left[f(y) \int b(y, z) g(z) d z+g(y) \int b(y, z) f(z) d z\right] .
$$

The first formula is a consequence of results obtained in Section 3 . The second formula can be obtained by a similar argument which counts the locations of pairs of particles. The details are spelled out in Sawyer (1976) (see (II.4)-(II.7), p. 686-7).

When $b(x, y)=b(y-x)$ these formulas can be simplified considerably. In this case

$$
E^{x} \eta_{t}(A)=\int d x m(t, x, A)=e^{-t} \sum_{n=0}^{\infty} \frac{t^{n}}{n !} \int d x b_{n}(x, A)=|A| .
$$

Using Formula (6.3) the expression for $w\left(t, A_{1}, A_{2}\right)$ may be rewritten as

$$
\begin{aligned}
& \frac{1}{2} \int_{0}^{t} d s \int d y m\left(s, y, A_{1}\right) \int b(z-y) m\left(s, z, A_{2}\right) d z \\
& +\frac{1}{2} \int_{0}^{t} d s \int d y m\left(s, y, A_{2}\right) \int b(z-y) m\left(s, z, A_{1}\right) d z .
\end{aligned}
$$

Since $m(s, y, B) \geqq 0$ for all $s, y, B$ it follows from (6.4) (or from (6.2)) that $E^{\mathscr{L}} \eta_{t}\left(A^{2}\right)$ increases as $t$ increases. The next result gives a necessary and sufficient condition for the limit to be finite.

Theorem 6.1. Let $A$ be a bounded open set. $\sup _{t \geq 0} E^{\mathscr{P}} \eta_{t}(A)<\infty$ if and only if the (discrete time) random walk which takes steps with distribution $b^{0}(y)=$ $[b(y)+b(-y)] / 2$ is transient.

Proof. Consider the first integral in (6.4) above. By making the substitution $v=z-y$ and interchanging the order of integration we can rewrite the expression as

$$
\frac{1}{2} \int d v b(v) \int_{0}^{t} d s \int d y m\left(s, y, A_{1}\right) m\left(s, y, A_{2}-v\right) .
$$

From formula (3.3) we can write

$$
m(s, y, A)=e^{-s} 1_{\{y \in A\}}+\int_{A} m_{0}(s, y, z) d z .
$$

Using formula (6.5) we can write

$$
\begin{aligned}
& \int \operatorname{dym}\left(s, y, A_{1}\right) m\left(s, y, A_{2}-v\right)=e^{-2 s}\left|A_{1} \cap\left(A_{2}-v\right)\right| \\
& \quad+e^{-s} \int_{A_{1}} d y \int_{A_{2}-v} d z_{2} m_{0}\left(s, y, z_{2}\right)+e^{-s} \int_{A_{1}} d z_{1} \int_{A_{2}-v} d y m_{0}\left(s, y, z_{1}\right) \\
& \quad+\int_{A_{1}} d z_{1} \int_{A_{2}-v} d z_{2} \int d y m_{0}\left(s, y, z_{1}\right) m_{0}\left(s, y, z_{2}\right) .
\end{aligned}
$$


It is easy to see that the integral from $s=0$ to $\infty$ of the sum of the first three terms in the last expression

$$
\leqq \frac{1}{2}\left|A_{1} \cap\left(A_{2}-y\right)\right|+\left|A_{1}\right|+\left|A_{2}-v\right|<\infty
$$

so $E^{\mathscr{L}} \eta_{t}(A)^{2} \uparrow \infty$ if and only if

$$
\int_{0}^{\infty} d s \int_{\mathrm{A}_{1}} d z_{1} \int_{\mathrm{A}_{2}-v} d z_{2} \int d y m_{0}\left(s, y, z_{1}\right) m_{0}\left(s, y, z_{2}\right)=\infty .
$$

Let $S_{n}$ be the sum of $n$ independent random variables with distribution given by $b(y)$ and $N_{s}$ be an independent Poisson random variable with mean $s$. It is easy to see that for any set $B$

$$
\int_{B} m_{0}(s, y, z) d z=P\left\{y+S_{N_{s}} \in B, N_{s} \geqq 1\right\} .
$$

If we let $m_{0}(s, x, y)=m_{0}(s, y, x)$ and $S_{n}^{\prime}, N_{s}^{\prime}$ be random variables which are independent of $S_{n}, N_{s}$ and have the same distribution then we can rewrite the expression in (6.6) as

$$
\begin{aligned}
\int_{0}^{\infty} d s \int_{\mathrm{A}_{1}} d z_{1} \int_{\mathrm{A}_{2}-v} d z_{2} \int d y m_{0}\left(s, z_{1}, y\right) m_{0}\left(s, y, z_{2}\right) \\
\quad=\int_{0}^{\infty} d s \int_{\mathrm{A}_{1}} d z_{1} P\left\{z_{1}+S_{\mathrm{N}_{\mathrm{s}}}-S_{\mathrm{N}_{\mathrm{s}}^{\prime}} \in A_{2}-v, N_{s} \geqq 1, N_{s}^{\prime} \geqq 1\right\} .
\end{aligned}
$$

The last expression is finite if and only if the random walk generated by $b^{0}(y)=[b(y)+b(-y)] / 2$ is transient so the proof is complete.

Using Theorem 6.1 we can now prove the first result mentioned in this section.

Theorem 6.2. If the random walk generated by $b^{\circ}(y)$ is transient then for each $t>0$ there is a non-trivial solution of $\Lambda T_{t}=\Lambda$ which is translation invariant.

Proof. Let $t>0$ and define a measure on $(M, M)$ by

$$
\mathscr{X}_{n}(A)=\frac{1}{n} \sum_{m=1}^{n} \mathscr{X} T_{m t}(A) .
$$

From Theorem 6.1 we have that if $K$ is bounded

$$
\sup _{m \geqq 1} E^{\mathscr{D}} \eta_{m t}(K)^{2}=C<\infty \text {. }
$$

This implies that

$$
\sup _{n \geqq 1} \int \mathscr{X}_{n}(d \eta) \eta(K)^{2}<\infty
$$

and hence that $\mathscr{X}_{n}$ has a subsequence $\mathscr{X}_{n_{k}}$ which converges weakly to a limit $\mathscr{X}_{\infty}$ (see Jagers (1974), p. 209). 
It is easy to see that $\mathscr{X}_{\infty}$ is translation invariant. To check that $\mathscr{X}_{\infty} T_{t}=\mathscr{X}_{\infty}$ observe that

$$
\mathscr{Q}_{n} T_{t}=\mathscr{X}_{n}+n^{-1}\left(\mathscr{X} T_{(n+1) t}-\mathscr{X}\right)
$$

so from (6.7) $\mathscr{X}_{n_{k}} T_{t} \Rightarrow \mathscr{X}_{\infty}$. Now $\mathscr{X}_{n_{k}} \Rightarrow \mathscr{X}_{\infty}$ and from results in Section $2 T_{t}$ has the Feller property, so it follows that $\mathscr{X}_{\infty} T_{t}=\mathscr{X}_{\infty}$.

To complete the proof we have to show that $\mathscr{L}_{\infty}$ is not the trivial point process. To do this we observe that if $K$ is a bounded set and $C$ is a positive integer

$$
\begin{aligned}
& \left|\int \eta(K) \mathscr{X}_{n}(d \eta)-\int \eta(K) \mathscr{X}_{\infty}(d \eta)\right| \\
& \leqq \\
& \quad\left|\int_{\{\eta(K) \leq C\}} \eta(K) \mathscr{X}_{n}(d \eta)-\int_{\{\eta(K) \leq C\}} \eta(K) \mathscr{X}_{\infty}(d \eta)\right| \\
& \quad+\frac{2}{C} \sup _{m \geq 1} \int \eta(K)^{2} \mathscr{X}_{m}(d \eta) .
\end{aligned}
$$

From (6.3) we have that $\int \eta(K) \mathscr{X}_{n}(d \eta)=|K|$ for all $n \geqq 1$ so letting $n \rightarrow \infty$ in the expression above gives

$$
|| K\left|-\int \eta(K) \mathscr{X}_{\infty}(d \eta)\right| \leqq \frac{2}{C} \sup _{m \geqq 1} \int \eta(K)^{2} \mathscr{X}_{m}(d \eta) .
$$

Since this holds for all $C, \int \eta(K) \mathscr{X}_{\infty}(d \eta)=|K|$ and so $\mathscr{X}_{\infty}$ is not the trivial point process.

Now that we have shown that solutions of $\Lambda T_{t}=\Lambda$ exist we can use results of Debes, Kerstan, Liemant and Matthes, which were proved for clustering operations on point processes, to characterize the class of translation-invariant stationary solutions of $\Lambda T_{t}=\Lambda$ which have finite intensity. From Theorems 4.3 and 4.4 in Matthes (1972) we get the following result.

Theorem 6.3. For every $t>0$ and $\rho \geqq 0$ there is exactly one ergodic translation-invariant point process $\Lambda_{t, \rho}^{*}$ with intensity $\rho$ which is stationary for $T_{t}$. The distributions $\Lambda_{t, \rho}^{*}, \rho \geqq 0$ form a semigroup, that is, if $\rho_{1}, \rho_{2} \geqq 0$ then $\Lambda_{t, \rho_{1}+\rho_{2}}^{*}$ is the distribution of a superposition of two independent point processes with distributions $\Lambda_{t, \rho_{1}}^{*}$ and $\Lambda_{t, \rho_{2}}^{*}$.

With this result it is easy to show that the family of stationary distributions is independent of $t$. To do this we observe that for all $n \geqq 0 \Lambda_{2^{-n}, \rho}^{*}$ is an ergodic translation-invariant point process with $\Lambda_{2^{-n}, \rho}^{*} T_{1}=\Lambda_{2^{-n}, \rho}^{*}$ so $\Lambda_{2^{-n, \rho}}^{*}=\Lambda_{1, \rho}^{*}$ and we have shown

$$
\Lambda_{1, \rho}^{*} T_{m 2^{-n}}=\Lambda_{1, \rho}^{*} \text { for all } m, n \geqq 0 .
$$

From (6.9) $\Lambda_{1, \rho}^{*} T_{t}$ has intensity $\rho$ for each $t$, so from the definition of $\eta_{t}$ it follows that $\Lambda_{1, \rho}^{*}\left\{\xi: t \rightarrow \eta_{t}^{\xi}\right.$ is right-continuous $\}=1$. Using (6.9) again we can 
conclude that for all $t \geqq 0 \Lambda_{1, \rho}^{*} T_{t}=\Lambda_{1, \rho}^{*}$ and hence $\Lambda_{t, \rho}^{*}=\Lambda_{1, \rho}^{*}$. This shows that there is one family of ergodic point processes which are stationary for all $t \geqq 0$.

From the work of Debes et al. (1970), pp. 241, 213, 199, we can also obtain a convergence theorem.

Theorem 6.4. If $\Lambda$ is a point process with an asymptotic density $\gamma(\eta)$ which has $\int \gamma(\eta) \Lambda(d \eta)<\infty$ then for all $t>0$

$$
\Lambda T_{n t} \Rightarrow \int \Lambda_{t, \gamma(\eta)}^{*} \Lambda(d \eta)
$$

From this result and Theorem 2.1 we can conclude that if $\Lambda T_{t}=\Lambda$ and $\Lambda$ is translation invariant with finite intensity then $\Lambda$ is representable as a mixture of the $\Lambda_{\rho}^{*}$.

7. $\beta=1, d=1$

In this section we shall show that when $\beta=1$ there are no non-trivial translation-invariant stationary distributions for the one-dimensional model when $\int|y| b(y) d y<\infty$. The proof of this result is based on the following result which is due to Liemant (see Matthes (1972), Theorem 4.3).

Theorem 7.1. The equation $\Lambda T_{t}=\Lambda$ has a non-trivial translation-invariant solution $\Lambda$ with finite intensity if and only if for some compact set $K$

$$
\inf _{n \geq 1} \int P\left\{\eta_{n t}^{x}(K)>0\right\} d x>0 .
$$

Proof. We shall first show the condition is necessary. To do this we observe that if $\Lambda$ has mean measure $\lambda d x$ then $\lambda \int P\left\{\eta_{n t}^{x}(K)>0\right\} d x$ is the expected number of particles at time 0 which have offspring in $K$ at time $n t$. Since

$$
P^{\Lambda}\left\{\eta_{n t}(K)>0\right\} \leqq \lambda \int P\left\{\eta_{n t}^{x}(K)>0\right\} d x
$$

it follows that if $\Lambda T_{t}=\Lambda$

$$
P^{\wedge}\{\eta(K)>0\}=\inf _{n \geq 1} P^{\wedge}\left\{\eta_{n t}(K)>0\right\}=0 .
$$

Since this holds for all compact sets $K$, the only translation-invariant solution of $\Lambda T_{t}=\Lambda$ with finite intensity is concentrated on the empty configuration.

To prove the converse we have to construct a translation-invariant equilibrium. Let $\mathscr{L}$ be a Poisson point in $R^{d}$ with intensity 1 and let

$$
\mathscr{X}_{n}=\frac{1}{n} \sum_{m=1}^{n} \mathscr{X} T_{m t}
$$


For each compact set $K \int \eta(K) \mathscr{X}_{n}(d \eta)=|K|<\infty$ so it follows from Jagers (1974), p. 209 that there is a subsequence of $\mathscr{X}_{n}$ which converges weakly to a limit $\mathscr{X}_{\infty}$.

It is easy to see that $\mathscr{L}_{\infty}$ is translation invariant. A proof given in Section 6 shows that $\mathscr{L}_{\infty} T_{t}=\mathscr{L}_{\infty}$ so to complete the proof we have to show that $\mathscr{X}_{\infty}$ is not the trivial point process. To do this we observe that the number of particles alive at time 0 which have offspring in $K$ at time $m t$ has a Poisson distribution with mean $\int P\left\{\eta_{m t}^{x}(K)>0\right\} d x$ so

$$
\mathscr{X} T_{m t}(\{\eta: \eta(K)=0\})=\exp \left(-\int P\left\{\eta_{m t}^{x}(K)>0\right\} d x\right) .
$$

From this if follows that

$$
\mathscr{X}_{n}(\{\eta: \eta(K)=0\})=n^{-1} \sum_{m=1}^{n} \exp \left(-\int P\left\{\eta_{m t}^{x}(K)>0\right\} d x\right)
$$

so if (7.1) holds

$$
\mathscr{X}_{\infty}(\{\eta: \eta(K)=0\}) \leqq \sup _{m} \mathscr{X}_{n}(\{\eta: \eta(K)=0\})<1
$$

and this completes the proof.

As a consequence of the proof given above we have the following result.

Corollary 7.2. Let $\mathscr{L}$ be a Poisson process with intensity 1 and suppose that for every compact set $K \mathscr{X} T_{n t}(\{\eta: \eta(K)>0\}) \rightarrow 0$ as $n \rightarrow \infty$. Then the equation $\Lambda T_{t}=\Lambda$ does not have a non-trivial translation-invariant solution.

Using Theorem 7.1 it is easy to show that there are no non-trivial translation-invariant stationary distributions when $d=1$ and $\int|y| b(y) d y<\infty$.

Theorem 7.3. If $d=1$ and $\int|y| b(y) d y<\infty$ then for every compact set $K$

$$
\lim _{t \rightarrow \infty} \int P\left\{\eta_{t}^{x}(K)>0\right\} d x=0 .
$$

Proof. It suffices to show that the result holds for all intervals of the form $[-a, a], a>0$. Let $\nu=\int y b(y) d y$ and $I_{\varepsilon, t}=[(\nu-2 \varepsilon) t,(\nu+2 \varepsilon) t]$

$$
\begin{aligned}
& \int P\left\{\eta_{t}^{x}(K)>0\right\} d x=\int_{I_{e . t}^{\mathrm{c}}} P\left\{\eta_{t}^{x}(K)>0\right\} d x \\
& +P\left\{\eta_{t}^{0} \neq \varnothing\right\} \int_{t^{c . t}} P\left\{\eta_{t}^{x}(K)>0 \mid \eta_{t}^{x} \neq \oslash\right\} d x \leqq \int_{I^{e}, t} E \eta_{t}^{x}(K) d x+4 \varepsilon t P\left\{\eta_{t}^{0} \neq \varnothing\right\}
\end{aligned}
$$

From formula (3.4)

$$
E \eta_{t}^{x}(K)=\sum_{n=0}^{\infty} e^{-t} \frac{t^{n}}{n !} b_{n}(x, K)
$$


If we let $S_{n}$ be the sum of $n$ independent random variables with distribution $b(y)$ and $N_{t}$ is an independent Poisson random variable with mean $t$ then

$$
E \eta_{t}^{x}(K)=P\left\{x+S_{N_{t}} \in K\right\}
$$

so

$$
\int_{-\infty}^{(-\nu-2 \varepsilon) t} E \eta_{t}^{x}(K) d x=\int_{-\infty}^{(-\nu-2 \varepsilon) t} P\left\{-x-a \leqq S_{N_{t}} \leqq-x+a\right\} d x .
$$

If $a<\varepsilon t$ and $x \leqq(-\nu-2 \varepsilon) t$ then $-x-a \geqq(\nu+\varepsilon) t$ so if $t>a / \varepsilon$

$$
\int_{-\infty}^{(-\nu-2 \varepsilon) t} E \eta_{t}^{x}(K) d x \leqq 2 a P\left\{S_{N_{t}} \geqq(\nu+\varepsilon) t\right\} .
$$

Now from the weak law of large numbers $N_{t} / t$ converges in probability to 1 and $S_{n} / n$ converges in probability to $\nu$ so $S_{N_{t}} / t$ converges in probability to $\nu$ and $P\left\{S_{N_{t}} \geqq(\nu+\varepsilon) t\right\} \rightarrow 0$. By a similar argument the integral over $((-\nu+2 \varepsilon) t, \infty)$ converges to 0 and so

$$
\int_{I_{\text {e.t. }}^{\mathrm{c}}} E \eta_{t}^{\mathrm{x}}(K) d x \rightarrow 0 .
$$

Now from Athreya and Ney (1972), p. 113, $t P\left\{\eta_{t}^{0} \neq \varnothing\right\} \rightarrow 2$ as $t \rightarrow \infty$ so from formula (7.3) it follows that

$$
\limsup _{t \rightarrow \infty} \int P\left\{\eta_{t}^{x}(K)>0\right\} d x \leqq 8 \varepsilon
$$

for all $\varepsilon>0$ which completes the proof.

Remark. The proof given above also shows that the same conclusion holds if the weak law of large numbers holds for the density $b$ in the form $S_{n} / n$ converges to $\nu$ in probability. This condition implies that the weak law of large numbers holds for the density $b^{0}$ in the form $S_{n}^{0} / n \rightarrow 0$, and this implies that the random walk which takes step according to $b^{0}$ is recurrent. No two of these conditions are equivalent.

8. $\beta=1, d=2$

In this section we shall show that when $\beta=1$ there are no non-trivial translation-invariant stationary distributions for the two-dimensional model when $\int|y|^{2} b(y) d y<\infty$. To simplify the cumbersome expressions below we shall suppose also that $\int y b(y) d y=0$ and that the determinant of the covariance matrix $\Sigma$ of $b(y)$ is 1 and leave it to the reader to check that the same proof works if $\nu$ and $\Sigma<\infty$ are arbitrary.

The method of proof is to show that if $\Lambda$ is a translation-invariant point 
process with intensity 1 and $A$ is a bounded open set with $|\partial A|=0$ then

$$
\lim _{t \rightarrow \infty} \frac{\log t}{8 \pi} P^{\Lambda}\left\{\frac{8 \pi}{\log t} \frac{\eta_{t}(A)}{|A|}>h\right\}=e^{-h}
$$

for all $h>0$. To see that this implies the desired result observe that if $\mathscr{X}$ is a Poisson process then from (6.3) $E^{\mathscr{C}} \eta_{t}(A)=|A|$ and from (8.1)

$$
\liminf _{t \rightarrow \infty} E^{\mathscr{L}}\left[\eta_{t}(A) ; \eta_{t}(A) \geqq \delta(\log t) / 8 \pi\right] \geqq|A| \int_{\delta}^{\infty} e^{-h} d h
$$

for each $\delta>0$ so for each $C<\infty$

$$
\limsup _{t \rightarrow \infty} E^{\mathscr{E}}\left[\eta_{t}(A) ; \eta_{t}(A) \leqq C\right]=0 .
$$

Using Corollary 7.2 now shows that if (8.1) holds then there are no non-trivial translation-invariant stationary distributions.

The rest of this section will be devoted to proving (8.1). To do this we shall begin by computing the moments of $\eta_{t}(A)$ in a branching random walk.

Theorem 8.1. If $A$ is a bounded open set with $|\partial A|=0$ then

$$
\lim _{t \rightarrow \infty} \frac{t}{(\log t)^{k-1}} E^{x}\left[\eta_{t}(A)\right]^{k}=\frac{k !|A|^{k}}{4^{k-1}(2 \pi)^{k}} .
$$

Proof. The proof is by induction. For $k=1$ the statement says

$$
\lim _{t \rightarrow \infty} \operatorname{tm}(y, x, A)=|A| / 2 \pi
$$

which is a consequence of (3.3) and the fact that $n b_{n}(x, A) \rightarrow|A| / 2 \pi$.

Suppose now that $k \geqq 2$ and that the statement holds for $j<k$. To prove that the result also holds for $k$ we will use the following formula.

Lemma 8.2. For all $k \geqq 1$

$$
\begin{aligned}
E^{x}\left[\eta_{t}(A)\right]^{k}=m(t, x, A)+\int d z b(z) \sum_{j=1}^{k-1} \frac{1}{2}\left(\begin{array}{l}
k \\
j
\end{array}\right) & \int_{0}^{t} d s \int m(t-s, x, d y) \\
& \times E^{y} \eta_{s}(A)^{j} E^{y+z} \eta_{s}(A)^{k-j} .
\end{aligned}
$$

This result is a special case of a formula derived in Appendix 1 of Fleischman (1978). The argument given there is an extension of the one given by Sawyer in the case $k=2$ (see Sawyer (1976), Appendix II).

To simplify the computations below it is desirable to reduce the size of expression (8.2). To do this we observe that if $k \geqq 2, \operatorname{tm}(t, x, A) /(\log t)^{k-1} \rightarrow 0$, so we can evaluate the limit of $t E^{x} \eta_{t}(A)^{k} /(\log t)^{k-1}$ by deriving an asymptotic formula for

$$
\int_{2}^{t} d s(\log s)^{k-2} \int m(t-s, x, d y) \frac{E^{y} \eta_{s}(A)^{j}}{(\log s)^{j-1}} \frac{E^{y+z} \eta_{s}(A)^{k-j}}{(\log s)^{k-j-1}} .
$$


To obtain the desired formula we shall show the following.

Lemma 8.3. Let $k_{1}(t, y, A)$ and $k_{2}(t, y, A)$ be functions which have the following three properties:

(i) $\lim _{t \rightarrow \infty} \int d y k_{i}(t, y, A)=1$;

(ii) there are some normal probability densities $\varphi_{i}$ such that $t k_{i}\left(t, y_{t}, A\right) \rightarrow$ $\varphi_{i}(y)$ whenever $y_{t} / t^{1 / 2} \rightarrow y$;

(iii) there are constants $B_{i}, C_{i}<\infty$ such that, for all $y \in R^{d}$

$$
\begin{gathered}
\sup _{t \geqq 1} \int d y k_{i}(t, y, A) \leqq B_{i} \\
\sup _{t \geqq s \geqq 1} \int m(t-s, y, d z) k_{i}(s, z, A) \leqq B_{i}
\end{gathered}
$$

and

$$
\sup _{s \geqq 1} s k_{i}(s, y, A) \leqq C_{i}
$$

Let $z \in R^{d}$ and let $l$ be a positive integer. If we define

$$
j(t, x, A)=\int_{2}^{t} d s(\log s)^{l-1} \int_{D} m(t-s, x, d y) k_{1}(s, y, A) k_{2}(s, y+z, A)
$$

for $t \geqq 2$ then it follows that

(a) $\lim _{t \rightarrow \infty} \int d x j(t, x, A) /(\log t)^{l}=l^{-1} \int \varphi_{1}(u) \varphi_{2}(u) d u$;

(b) if $x_{t} / t^{1 / 2} \rightarrow x$ then

$$
\lim _{t \rightarrow \infty} t j\left(t, x_{t}, A\right) /(\log t)^{l}=l^{-1} \varphi(x) \int \varphi_{1}(v) \varphi_{2}(v) d v
$$

where $\varphi(x)=\lim _{t \rightarrow \infty} \operatorname{tm}\left(t, x_{t},[0,1]^{d}\right)$;

and

(c) there are constants $B_{0}, C_{0}<\infty$ such that, for all $x \in R^{d}$

$$
\begin{gathered}
\sup _{t \geqq 2} \int d x j(t, x, A) /(\log t)^{l} \leqq B_{0} \\
\sup _{t \geqq s \geqq 2} \int t-\operatorname{sm}(t-s, x, d y)\left[j(s, y, A) /(\log s)^{l}\right] \leqq B_{0}
\end{gathered}
$$

and $\sup _{t \geqq 2} t\left[j(t, x, A) /(\log t)^{l}\right] \leqq C_{0}$.

Proof. Let $z \in R^{d}$ and let

$$
\begin{gathered}
I_{t}^{x}(u, v)=\int_{u}^{v} d s(\log s)^{l-1} \int m(t-s, x, d y) k_{1}(s, y, A) k_{2}(s, y+z, A) \\
J_{t}(u, v)=\int d x I_{t}^{x}(u, v)=\int_{u}^{v} d s(\log s)^{l-1} \int d y k_{1}(s, y, A) k_{2}(s, y+z, A)
\end{gathered}
$$


By hypothesis $\int d y k_{1}(s, y, A) k_{2}(s, y+z, A) \leqq B_{1} C_{2} s^{-1}$ so if $\varepsilon \geqq 2 t^{-1}$

$$
J_{t}(t \varepsilon, t) \leqq B_{1} C_{2} \int_{t \varepsilon}^{t} d s(\log s)^{l-1} s^{-1}=B_{1} C_{2} l^{-1}\left[(\log t)^{1}-(\log t \varepsilon)^{l}\right] .
$$

From (8.3) it follows that if $\log \varepsilon_{t} / \log t \rightarrow 0$ then $\left(\log \left(t \varepsilon_{t}\right) / \log t\right)^{l} \rightarrow 1$ and $J_{t}\left(t \varepsilon_{t}, t\right) /(\log t)^{l} \rightarrow 0$.

To trim off the other end we pick $s_{t} \uparrow \infty$ such that $s_{t} /(\log t)^{l} \rightarrow 0$. From the observation above we have

$$
J_{t}\left(2, s_{t}\right) \leqq \int_{2}^{s_{t}} d s(\log s)^{l-1} s^{-1} B_{1} C_{2} \leqq B_{1} C_{2} s_{t}\left(\log s_{t}\right)^{l-1_{2}-1}
$$

so under our choice of $s_{t} J_{t}\left(2, s_{t}\right) /(\log t)^{1} \rightarrow 0$.

Let $K_{s}$ be an increasing function of $s$ which has $\lim _{s \rightarrow \infty} K_{s}=\infty$. Let $D_{s}=$ $\left\{y:|y| \leqq K_{s} s^{1 / 2}\right\}$. Under assumption (iii) we have

$$
\begin{aligned}
(\log t)^{-l} \int_{s_{t}}^{t \varepsilon_{t}} d s(\log s)^{l-1} s^{-1} \int_{D_{s}^{c}} d y k_{1}(s, y, A) s k_{2}(s, y+z, A) & \\
& \leqq \sup _{s \geq s_{t}} \int_{D_{s}^{c}} d y k_{1}(s, y, A) C_{2} .
\end{aligned}
$$

It follows from (i) and (ii) that the right-hand side of (8.5) converges to 0 as $t \rightarrow \infty$ so the main contribution to $J_{t}\left(s_{t}, t \varepsilon_{t}\right)$ comes from integrating over the sets $D_{s}$. To evaluate this piece of the integral we observe that

$$
s \int_{D_{s}} k_{1}(s, y, A) k_{2}(s, y+z, A) d y=\int_{s^{-1 / 2} D_{s}} s k_{1}\left(s, u s^{1 / 2}, A\right) s k_{2}\left(s, u s^{1 / 2}+z, A\right) d u
$$

so it follows from (i) and (ii) that there are $K_{\mathrm{s}} \uparrow \infty$ which increase to $\infty$ slowly enough so that

$$
s \int_{D_{s}} k_{1}(s, y, A) k_{2}(s, y+z, A) d y \rightarrow \int \varphi_{1}(u) \varphi_{2}(u) d u .
$$

From this it follows that

$$
\begin{aligned}
\lim _{t \rightarrow \infty} \int_{s_{i}}^{t \varepsilon_{i}} d s \frac{(\log s)^{l-1}}{s} \int_{D_{s}} d y k_{1}(s, y, A) s k_{2}(s, y+z, A) & \\
& =\int \varphi_{1}(u) \varphi_{2}(u) d u .
\end{aligned}
$$

Combining formulas (8.3)-(8.6) now proves conclusion (a).

To prove (b) we observe that from hypothesis (iii)

$$
\int_{D} \operatorname{tm}(t-s, x, d y) k_{1}(s, y, A) \leqq\left\{\begin{array}{lll}
2 B_{1} & \text { for } & s \leqq t / 2 \\
2 C_{1} & \text { for } & s \geqq t / 2
\end{array}\right.
$$


so if $\varepsilon \geqq 2 t^{-1}$ then by the argument given for (8.3)

$$
t I_{t}(t \varepsilon, t) \leqq 2\left(B_{1}+C_{1}\right) C_{2} l^{-1}\left[(\log t)^{l}-(\log t \varepsilon)^{l}\right] .
$$

From (8.7) it follows that if $\log \varepsilon_{t} / \log t \rightarrow 0$ then $t I_{t}\left(t \varepsilon_{t}, t\right) /(\log t)^{l} \rightarrow 0$. In the same way we can conclude that if $s_{t} /(\log t)^{l} \rightarrow 0$ then

$$
\lim _{t \rightarrow \infty} t I_{t}\left(2, s_{t}\right) /(\log t)^{l}=0 .
$$

Let $K_{s} \uparrow \infty$ and $D_{s}=\left\{y:|y| \leqq K_{s} s^{1 / 2}\right\}$. It follows from the local central limit theorem and hypotheses (i)-(iii) that if $\delta>0$ then

$$
\begin{array}{rl}
(\log t)^{-l} \int_{s_{t}}^{t \varepsilon_{t}} d s(\log s)^{l-1,-1} \int_{D_{s}^{c}} & t m\left(t-s, x_{t}, d y\right) k_{1}(s, y, A) s k_{2}(s, y+z, A) \\
\leqq & l^{-1}\left(\frac{\log t \varepsilon_{t}}{\log t}\right)^{l} \delta C_{2} \quad \text { for } t \text { sufficiently large. }
\end{array}
$$

So again the main contribution comes from the integral over $D_{s}$.

To evaluate the other integral we observe that if $s \leqq t \varepsilon_{t}$ then $D_{s}$ has radius $K_{s} s^{1 / 2} \leqq K_{t \varepsilon_{t}}\left(t \varepsilon_{t}\right)^{1 / 2}$ so if $t^{-1 / 2} K_{t \varepsilon_{t}}\left(t \varepsilon_{t}\right)^{1 / 2} \rightarrow 0$ then it follows from (8.6) and the local central limit theorem that whenever $x_{t} / t^{1 / 2} \rightarrow x$,

$$
\begin{array}{rl}
\lim _{t \rightarrow \infty}(\log t)^{-l} \int_{s_{t}}^{t \varepsilon_{t}} & d s(\log s)^{l-1} s^{-1} \int_{D_{s}} t m\left(t-s, x_{t}, d y\right) \\
& \times k_{1}(s, y, A) s k_{2}(s, y+z, A)=\varphi(x) \int \varphi_{1}(u) \varphi_{2}(u) d u
\end{array}
$$

where $\varphi(x)=\lim _{t \rightarrow \infty} \operatorname{tm}\left(t, x_{t},[0,1]^{d}\right)$.

Combining formulas (8.7)-(8.10) proves conclusion (b).

To complete the proof of the lemma we have to show that the conclusions in (iii) hold. To prove the first we observe that

$$
\begin{aligned}
\int d x j(t, x, A) /(\log t)^{l} \\
\quad=(\log t)^{-l} \int_{2}^{t} d s(\log s)^{l-1} s^{-1} \int d y k_{1}(s, y, A) s k_{2}(s, y+z, A) \leqq B_{1} C_{2} l^{-1} .
\end{aligned}
$$

To prove the second we write

$$
\begin{aligned}
\int(t-s) m(t-s, w, d x) j(t, x, A) & =\int_{2}^{s} d u(\log u)^{l-1} \int t-s m(t-s, w, d x) \\
& \times \int^{2} m(s-u, x, d y) k_{1}(u, y, A) k_{2}(u, y+z, A) .
\end{aligned}
$$

Since $t-s \geqq t-u$ over the range of integration this shows

$$
\int(t-s) m(t-s, w, d x) j(t, x, A) /(\log t)^{l} \leqq B_{1} C_{2} l^{-1} .
$$


To prove the third inequality we observe that from the proof of (8.7)

$$
t j(t, x, A) /(\log t)^{l} \leqq 2\left(B_{1}+C_{1}\right) C_{2} l^{-1} .
$$

The proof of Lemma 8.3 is now complete. To finish the proof of Theorem 8.1 it suffices to show that the indicated constants arise when Lemma 8.3 is applied to formula (8.2)

Let

$$
v_{k}=\lim _{t \rightarrow \infty} \frac{t}{(\log t)^{k-1}} \frac{E\left[\eta_{t}(A)\right]^{k}}{|A|^{k}} .
$$

We have already shown that $v_{1}=1 / 2 \pi$. From Lemmas 8.2 and 8.3 ((a) and (b)) it follows that for $k \geqq 2$

and so

$$
v_{k}=\frac{1}{2(k-1)} \sum_{j=1}^{k-1}\left(\begin{array}{l}
K \\
j
\end{array}\right) \varphi(0) 2 \pi v_{j} 2 \pi v_{k-j} / 4 \pi=\frac{1}{4(k-1)} \sum_{j=1}^{k-1}\left(\begin{array}{l}
k \\
j
\end{array}\right) v_{j} v_{k-j}
$$

$$
\frac{v_{k}}{k !} \frac{1}{4(k-1)} \sum_{j=1}^{k-1} \frac{v_{j}}{j !} \frac{v_{k-i}}{k-j !}
$$

Substituting $u_{k}=4^{k-1}(2 \pi)^{k} v_{k} / k$ ! in the last formula gives

$$
u_{k}=\frac{1}{(k-1)} \sum_{j=1}^{k-1} u_{i} u_{k-j} \text {. }
$$

Since $u_{1}=1$ it follows that $u_{k}=1$ for all $k$ and hence that $v_{k}=k ! / 4^{k-1}(2 \pi)^{k}$. This completes the proof of Theorem 8.1.

The next result gives an asymptotic formula for the moments of $\eta_{t}(A)$ in the infinite-particle system.

Theorem 8.4. If $\Lambda$ is a translation-invariant initial distribution with intensity 1 then

$$
\lim _{t \rightarrow \infty}(\log t)^{1-k} E^{\Lambda}\left[\eta_{t}(A)\right]^{k}=k !|A|^{k} /(8 \pi)^{k-1} .
$$

Proof. Under the hypothesis on $\Lambda, E^{\Lambda} \eta_{t}(A)=\int d x m(t, x, A)=|A|$ so the result holds trivially when $k=1$.

To obtain the result for $k \geqq 2$ we will use the results of Lemma 8.3. If $k=2$

$$
E\left[\eta_{t}^{\xi}(A)\right]^{2}=\sum_{x, y \in \xi} E\left[\eta_{t}^{x}(A) \eta_{t}^{y}(A)\right] .
$$

When $x \neq y, \eta_{t}^{x}(A)$ and $\eta_{t}^{y}(A)$ are independent so

$$
\begin{aligned}
E\left[\eta_{t}^{\xi}(A)\right]^{2}=\int \xi(d x) E\left[\eta_{t}^{x}(A)\right]^{2}+\int \xi(d x) E \eta_{t}^{x}(A) & \int \xi(d y) E \eta_{t}^{y}(A) \\
& -\int \xi(d x)\left[E \eta_{t}^{x}(A)\right]^{2}
\end{aligned}
$$

and $E^{\Lambda}\left[\eta_{t}(A)\right]^{2}=\int d x E\left[\eta_{t}^{x}(A)\right]^{2}+1-\int d x\left[E \eta_{t}^{x}(A)\right]^{2}$ 
From the proof of Lemma 8.3 there is a constant $C$ such that

$$
(\log t)^{-1} \int d x\left[E \eta_{t}^{x}(A)\right]^{2} \leqq C(\log t)^{-1} \int d x E \eta_{t}^{x}(A)=C|A|(\log t)^{-1} .
$$

This implies

$$
\lim _{t \rightarrow \infty} \frac{E^{\Lambda}\left[\eta_{t}(A)\right]^{2}}{\log t}=\lim _{t \rightarrow \infty} \int d x \frac{E\left[\eta_{t}^{x}(A)\right]^{2}}{\log t} .
$$

From conclusion (a) of Lemma 8.3 it now follows that

$$
\lim _{t \rightarrow \infty} \frac{E^{\Lambda}\left[\eta_{t}(A)\right]^{2}}{(\log t)}=1 \cdot \frac{1}{(2 \pi 2)}|A|^{2}=\frac{2|A|^{2}}{8 \pi} \text {. }
$$

To prove the result for $k \geqq 3$ we observe that by a similar argument

$$
\lim _{t \rightarrow \infty} \frac{E^{\Lambda}\left[\eta_{t}(A)\right]^{k}}{(\log t)^{k-1}}=\lim _{t \rightarrow \infty} \int d x \frac{E\left[\eta_{t}^{x}(A)\right]^{k}}{(\log t)^{k-1}}
$$

so it follows from Theorem 8.1 and conclusions (a) and (b) of Lemma 8.3 that

$$
\lim _{t \rightarrow \infty} \frac{E^{\Lambda}\left[\eta_{t}(A)\right]^{k}}{(\log t)^{k-1}}=(2 \pi) \frac{k !|A|^{k}}{4^{k-1}(2 \pi)^{k}} .
$$

This completes the proof of Theorem 8.4.

Having derived an asymptotic formula for the moments of $\eta_{t}(A)$ the next step in proving (8.1) is to use Theorems 8.1 and 8.4 to show the following.

Theorem 8.5. For all $\theta>0$

$$
\begin{gathered}
\lim _{t \rightarrow \infty} \frac{(t \log t)}{4}\left(1-E^{x} \exp \left(-8 \pi \theta \eta_{t}(A) /|A| \log t\right)\right)=\theta /(\theta+1) \\
\lim _{t \rightarrow \infty} \frac{\log t}{8 \pi}\left(1-E^{\Lambda} \exp \left(-8 \pi \theta \eta_{t}(A) /|A| \log t\right)\right)=\theta /(\theta+1) .
\end{gathered}
$$

Proof. We shall only prove the second result since it is the one which is needed to complete the derivation of (8.1). The idea of the proof is simple: if $\theta$ is 'sufficiently small' then

$$
E^{\Lambda}\left(\exp \left(-\theta \eta_{t}(A)\right)=1+\sum_{k=1}^{\infty} \frac{(-\theta)^{k}}{k !} E^{\Lambda}\left[\eta_{t}(A)\right]^{k}\right.
$$

and so

$$
(\log t) E^{\Lambda}\left[1-\exp \left(-\theta \eta_{t}(A) / \log t\right)\right]=-\sum_{k=1}^{\infty} \frac{(-\theta)^{k}}{k !} \frac{E^{\Lambda}\left[\eta_{t}(A)\right]^{k}}{(\log t)^{k-1}} .
$$

Now as $t \rightarrow \infty$ each term in the sum above converges to $(-\theta)^{k}|A|^{k} /(8 \pi)^{k-1}$. 
This suggests that as $t \rightarrow \infty$

$$
\frac{\log t}{8 \pi} E^{\Lambda}\left[1-\exp \left(\frac{-8 \pi \theta \eta_{t}(A)}{|A| \log t}\right)\right] \rightarrow-\sum_{k=1}^{\infty}(-\theta)^{k}=\frac{\theta}{1+\theta} .
$$

To prove this statement we have to prove that the sum and limit may be interchanged. To do this we observe that it follows from (8.2) that for all $|\theta|<8 \pi /|A|$ there is a constant $C_{\theta}<\infty$ such that

$$
\frac{\theta^{k}}{k !} \frac{E^{\Lambda}\left[\eta_{t}(A)\right]^{k}}{(\log t)^{k-1}} \leqq C_{\theta} \quad \text { for all } k \geqq 1 .
$$

This shows that the convergence in (8.13) occurs for all $\theta \in(-8 \pi /|A|, 8 \pi /|A|)$.

To complete the proof we have to show that the convergence occurs for $\theta \geqq 8 \pi /|A|$. To do this we let

$$
\varphi_{t}(\theta)=(\log t) E^{\Lambda}\left[1-\exp \left(-\theta \eta_{t}(A) / \log t\right)\right]
$$

for all complex $\theta$ with $\operatorname{Re} \theta>0$. The functions $\varphi_{t}(\theta)$ are analytic on the right half-plane and have $\left|\varphi_{t}(\theta)\right| \leqq 1$ when $\operatorname{Re} \theta>0$. It now follows from Vitali's theorem (see Hille (1962), Theorem 15.3.1) that $\varphi_{t}(\theta) \rightarrow \theta /(\theta+1)$ for all $\theta$ with $\operatorname{Re} \theta>0$. This shows that formula (8.13) is valid.

Using formulas (8.12) and (8.13) we can now show that for all $h>0$

$$
\begin{aligned}
& \lim _{t \rightarrow \infty} \frac{t \log t}{4} P^{x}\left\{\frac{8 \pi}{\log t} \frac{\eta_{t}(A)}{|A|}>h\right\}=e^{-h} \\
& \lim _{t \rightarrow \infty} \frac{\log t}{8 \pi} P^{\Lambda}\left\{\frac{8 \pi}{\log t} \frac{\eta_{t}(A)}{|A|}>h\right\}=e^{-h} .
\end{aligned}
$$

To do this let $X_{t}=8 \pi \eta_{t}(A) /|A| \log t, F_{t}(h)=P\left\{X_{t} \leqq h\right\}$. By Fubini's theorem

$$
\int_{0}^{\infty}\left(1-e^{-\theta h}\right) d F_{t}(h)=E \int_{0}^{X_{t}} \theta^{-1} e^{-\theta y} d y=\int_{0}^{\infty} \theta^{-1} e^{-\theta y} P\left\{X_{t}>y\right\} d y
$$

so

$$
\lim _{t \rightarrow \infty} \int_{0}^{\infty} e^{-\theta h} \frac{\log t}{8 \pi} P\left\{X_{t}>h\right\} d h=\theta^{-1} \lim _{t \rightarrow \infty} \frac{\log t}{8 \pi}\left(1-E^{\Lambda} e^{-\theta X_{t}}\right)=1 /(\theta+1) .
$$

\section{References}

Asmussen, S. and Kaplan, N. (1976) Branching random walks I. Stoch. Proc. Appl. 4, 1-13.

Athreya, K. AND Ney, P. (1972) Branching Processes. Springer-Verlag, Berlin.

BUHLER, W. J. (1970) The distribution of generations and other aspects of the family structure of a branching process. Proc. 6th Berkeley Symp. Math. Statist. Prob. 3, 463-480.

Daley, D. J. AND Vere-Jones, D. (1972) A summary of point processes. In Lewis (1972), 463-480.

Debes, H., Kerstan, J., Liemant, A. AND Matthes, K. (1970) Verallgemeinerungen eines Satzes von Dobrushin, I. Math. Nachr. 47, 183-224. 
Dobrushin, R. L. (1956) On Poisson laws for the distribution of particles in space. Ukrain. Math. Z. 8, 127-134.

Doob, J. (1953) Stochastic Processes. Wiley, New York.

Dunford, N. (1951) An individual ergodic theorem for noncommutative transformations. Acta Sci. Math. (Szeged) 14, 1-14.

Fleischman, J. (1978) Limiting distributions for branching random fields. Trans. Amer. Math. Soc. 239, 353-390.

Harris, T. (1963) The Theory of Branching Processes. Springer-Verlag, Berlin.

Harris, T. (1976) On a class of set-valued Markov processes. Ann. Prob. 4, 175-194.

HarRis, T. (1978) Additive set-valued Markov processes and percolation methods. Ann. Prob. 6, 355-378.

Hulle, E. (1962) Analytic Function Theory, Vol. II. Ginn, New York.

Holley, R. AND STROOK, D. (1978) Nearest neighbour birth and death processes on the real line. Acta Math. 140, 103-154.

JAGERS, P. (1974) Aspects of random measures and point processes. Advances in Probability 3, ed. P. Ney and S. Port, Dekker, New York, 179-239.

JofFE, A. AND MoncAYO, A. (1973) Random variables trees and branching random walks. Adv. Maths 10, 401-416.

Kallenberg, O. (1977) Stability of critical cluster fields. Math. Nachr. 77, 7-43.

Kaplan, N. AND Asmussen, S. (1976) Branching random walks II. Stoch. Proc. Appl. 4, 15-31.

KhaRmalov, B. P. (1968) On properties of branching processes with an arbitrary set of particle types. Theory Prob. Appl. 13, 84-98.

Lewis, P. A. W. (ed.) (1972) Stochastic Point Processes: Statistical Analysis, Theory, and Applications. Wiley, New York.

MATTHES, K. (1972) Infinitely divisible point processes. In Lewis (1972), 384-404.

NEY, P. (1965) The convergence of a random distribution function associated with a branching process. J. Math. Anal. Appl. 12, 316-327.

SAMuels, M. L. (1971) Distribution of the branching process population among generations. $J$. Appl. Prob. 8, 655-667.

SAWYER, S. (1976) Branching diffusion processes in population genetics. Adv. Appl. Prob. 8, $659-689$.

Smythe, R. (1976) Multiparameter subadditive processes. Ann. Prob. 4, 772-782.

Stone, C. (1965) On local and ratio limit theorems. Proc. 5th Berkeley Symp. Math. Statist. Prob. 2, 217-224.

Stone, C. (1968) On a theorem of Dobrushin. Ann. Math. Statist. 39, 1391-1401.

Sudbury, A. (1977) Clumping effects in models of isolation by distance. J. Appl. Prob. 14, 391-395. 